\title{
Excitons and their equilibration
}

\author{
R. S. Knox \\ Department of Physics and Astronomy and Rochester Theory Center for Optical \\ Science and Engineering, University of Rochester, Rochester, NY 14627-0171,USA
}

\begin{abstract}
A very selective survey of exciton research is presented, with a focus on certain processes of relaxation, dephasing, and equilibration that occur during the exciton lifetime. One of these is Förster transfer, which can be viewed as either dephasing or equilibration. An old theorem of Kennard and Stepanov is discussed in a new context and proposed as a possible diagnostic tool for some exciton spectra.
\end{abstract}

\section{INTRODUCTION}

Exciton studies have progressed through many stages that correspond to those in atomic studies, including electronic structure, interactions with other particles, determinations of oscillator strengths and ionization rates, bonding into excitonic molecules, condensation, and thermal equilibration. I will not try to provide full references here. Most of the general topics to be discussed can be found documented in my reviews $(1-4)$.

Driven by huge advances in computation and experimental techniques, exciton research has essentially evolved from a study of structures to a study of dynamics. In the very earliest work dynamics played an important role, but largely in connection with optical widths. In condensed matter physics, where the material structures were generally well known, the exciton's electronic structure was deduced in some detail rather early. The work of the 1950's and '60's could not benefit from the time resolution that we now consider routine, but a remarkable amount of dynamical information was obtained from steady-state spectra.

For lack of structural information, a parallel development for excitons in photosynthetic materials was not possible. The exciton, regarded primarily as a perturbed molecular excitation, was for many years used as a tool to infer the general structure of light-gathering systems of chlorophyll, carotenoids, phycobilins, and other important chromophores, often by using its diffusive (dynamical) properties as much as its optical properties. While modern technology has brought condensed-matter exciton dynamics into prominence, it has at the same time produced biological structural information sufficiently detailed that theories of exciton dynamics in biosystems can now be tested and challenged. Although the domains over which the exciton moves are not large and do not usually have high symmetry, protein structures containing on the order of dozens of chromophores can be studied from an ensemble viewpoint. This is due to the fact that large numbers of identical samples are produced genetically.

As a metastable object, the exciton has no chance of reaching a condition of true thermal equilibrium. This is particularly true of the excitons in photosynthetic cells where a substantial optical transition probability is required. However, when exciton lifetimes are long compared with scattering times, a quasiequilibration can be established. In many contexts the word 'relaxation' is used for the processes leading toward equilibration, and I may use the two words rather interchangeably, making some distinction when necessary.

I begin with a review of exciton research from a very personal perspective, and then discuss two examples of exciton equilibration. The first will be an alternative description of what is better known as excitation transfer, and the second will be a new view of a rather old theorem that is not encountered in most discussions of excitons. 


\section{A PERSONAL EXCITON PERSPECTIVE}

\section{The rare gas solids}

My involvement with the exciton's structure began with the simplest crystal (argon) and the oldest exciton (Frenkel's). Dexter, noting a suggestion of Peierls, felt that the rare gas solid would prove to be an ideal testing ground for the tight-binding model. None of us had considered the possibility that the $4 \mathrm{~s}$ electron would have a huge radius, and when the nearest-neighbor $4 \mathrm{~s}-4 \mathrm{~s}$ overlap integral turned out to be 0.55 , the test had a definitive conclusion. wave-function orthogonalization would be critical for application of the Frenkel model, and a standard overlap expansion could not be proved convergent. It was largely good luck that my truncated tight-binding calculation of the excitation energy was only about $20 \%$ too low. Shortly thereafter, Franco Bassani and I calculated the semiconductor-like conduction band of argon and Giancarlo Baldini discovered the bound-exciton hydrogenic series in Ar:Xe. Despite this massive change in the microscopic picture of solid argon's excitonic structure, its principal absorption bands remained close to their atomic, and therefore Frenkel-like, positions! This experience with rather diverse models operating in a single system tended to reinforce both my conservative and my radical views of exciton physics.

\section{The miniexciton, energy transfer, and nhotohiology}

Researchers in condensed matter will probably recognize 'miniexciton' as a term relating to a pair of dopants in an organic crystal. It is also appropriate for the special pair in photosynthesis, which consists of two closely-associated chlorophyll or bacteriochlorophyll molecules. The special pair's states generally lie lower than those of the surrounding chromophores and act as a trap for exciton energy. Many features of photosynthetic systems essentially involve exciton states for systems in which $N=2$. For example, energy transfer has been understood for nearly fifty years in terms of pairwise interacting chromophores (5). In a larger array known as the photosynthetic unit, excitons diffuse among $N=50$ to 200 chromophores and appear to obey an exciton diffusion equation. It is now recognized that in many cases, the rate of trapping of excitation by the special pair is relatively slow compared with the diffusion to the trap, leading to what has been termed transfer equilibrium (for a review, ref. 6).

Most light-harvesting chromophores are held in rigid specific arrays by protein binding. One clear function of such arrays is to enable energy transfer without strong spectral disturbance. In ordinary green plants, approximately 12 chlorophylls are found in the smallest functioning unit, and they have no obvious spatial symmetry (7). Highly symmetric ring-like structures $(N=8$ to 20 ) have been determined by X-ray diffraction in bacterial photosynthetic systems (e. g., ref. 8). Here, with strongly interacting transition moments, a delocalized exciton is clearly the best excited-state description. Bacterial light-gathering systems make use of these rings in a manner that is not yet completely clear. From the viewpoint of the exciton theorist all of these arrays pose large challenges; one can depend on neither large nor small $N$ to simplify a calculation. Symmetry helps, of course, in the case of the rings.

With this lengthy introduction, I return briefly to personal aspects. Having been closely associated with Dexter, my interest in the Förster transfer theory was rather high, and upon beginning to study photosynthesis I discovered that there were various problems of interpretation in connection with energy transfer (reviewed in part in refs. 3 and 9). One of these problems involved determining a rate of transfer between members of a chlorophyll pair as a function of coupling strength. The prevailing theories, one based on simple time evolution by quantum mechanics and one based on transition probabilities, led to different predicted rates. They were proportional to the coupling matrix element $V$ and to $V^{2}$, respectively, and the two were otherwise difficult to compare.

Here was a second example of diverse models being applied to a single phenomenon, this time in the dynamical rather than the structural domain. The apparent existence of two different mechanisms, combined with the absence of structural information, had resulted in considerable controversy (9). Kenkre and Rahman and I found the links between these (10), links that were to some extent known in other contexts but which I believe are only now proving important or at least useful in the interpretation of ultrafast miniexciton phenomena in photobiology. I will return to this in a later section. 


\section{Semiconductors and quantum wells}

When confined to a narrow range in one dimension, the semiconductor exciton becomes pancake-like and is built from similarly-constrained electron and hole states. Its oscillator strength is enhanced; such confined excitons are the object of great practical interest at high densities. Femtosecond spectroscopy is now capable of probing the relaxation of excitons such as these at very short times. Nonlinear exciton processes in quantum wells have, among others, the effect of modulating the well's optical properties, and this has led to the new field of femtosecond excitonic optoelectronics (11).

My own direct quantum-well exciton involvement has been with the interpretation of an observed transfer of excitation between widely-separated wells (12). As in the case of two molecules at several nanometer separation, the quantum wells may interact via transition-dipole resonance and thereby exchange energy in principle without exchanging charge carriers. The data appear consistent with a Förster-type resonance transfer process combined with momentum conservation transverse to the growth axis of the wells.

While quantum well structures and the photosynthetic chlorophyll-proteins might appear to have little in common, parallels for excitons are numerous: for both, the number of individual coupled entities $N$ is in the range 1-100; both involve intercommunication by tunneling and excitation transfer; both exhibit interesting nonlinear effects at high excitation intensity; and both have geometric uniformity, one by human design and the other by genetic control.

\section{EQUILIBRATION I: ENERGY TRANSFER AND DEPHASING}

\section{Eörster transfer as exciton equilibration}

Frenkel and Peierls considered exciton motion in rather general terms, developing two limiting cases: scattering and diffusion. Davydov approached excitons in organic crystals largely from the scattering viewpoint, concentrating on spectral effects of the molecular interactions. In the diffusion limit, which has until recently been the near-exclusive working model in photosynthesis, Förster (5b) was among the first to write a diffusion equation for the exciton. It was based on a quantitative computation of site-to-site transfer, thus, let us consider the miniexciton case of $N=2$.

The question Förster asked and answered was "what is the rate of transfer of excitation from an excited molecule to an identical neighbor initially in its ground state?" For close neighbors with strong dipole transitions, the rate he found was in the inverse-picosecond range (I use currently accepted rates in this discussion). Therefore, within the fluorescence lifetime, there is ample time for the pair to acquire equal shares of the excitation probability. This can be considered, and has been called, "transfer equilibration." Whereas identical molecules will end up with equal shares of excitation probability, unlike molecules can also undergo these processes, ending up with shares proportional to a Boltzmann factor $\exp \left(-F_{i} / k_{\mathrm{B}} T\right)$, where $F_{i}$ is the ground-to-excited-state free energy difference for molecule $i$ (unpublished work: P. D. Laible, R. S. Knox, and T. G. Owens). In a collection of $N>2$ close-lying chromophores, such a transfer equilibration is believed to be a rather general phenomenon in photosystem II of higher plants (13).

Förster originally applied his theory to the interpretation of fluorescence depolarization in dilute solutions, where transfer within a single two-chromophore pair is the dominant process. My colleagues and I (10b) noted a paradox in this application to depolarization. Immediately upon excitation, the transition moment of the pair should be oriented such that its projection on the exciting-light polarization vector is maximized, which requires consideration of the delocalized states of the pair, at least momentarily. The ensuing small correction to steady-state depolarization was considered negligible, but buried in it was an effect for future discovery by time-resolved spectroscopy.

\section{How localization might occur in a pair of identical molecules}

Considering now the delocalized eigenstates $| \pm\rangle$ of the $N=2$ system, we ask how excitation can be prepared on a single one of the sites. Having the polarization of one member of that site be parallel to the $E$ vector of the light is only part of the answer, because $E$ has a projection on both eigenstates of the pair. 
Since both eigenstates are delocalized, any excitation instantly produces probability on both sites. The solution of the problem is closer at hand if we assume that the states $| \pm\rangle$ are produced coherently. Then, it is possible that the otherwise neglected off-diagonal density matrix elements $\rho_{+-}$and $\rho_{-+}$will also be produced by the excitation in the geometry under consideration. With the help of these off-diagonal matrix elements, it is easily shown (10b) that a purely site-selected population can exist, with $\rho_{11}=1$, $\rho_{22}=0$.

As the coherences $\rho_{+-}$and $\rho_{-+}$decay, the probabilities of occupation of the two sites in the equivalent pair approach 0.5. This is precisely what happens in the Förster depolarization calculation and is therefore clearly an alternative way of describing the Förster energy transfer process: dephasing of the pair's miniexciton states. In order to ensure observable nonzero initial values of the coherences, very-shortpulse excitation broad enough to excite the two eigenstates coherently is required (14). The rationalization of this "eigenstate view" for steady-state excitation of ordinary pairs in solution appears not to have been discussed.

\section{Observing coherence in the $N=2$ svstem}

Anisotropy is now generally observed in time-resolved fluorescence or transient absorption (pump-probe) experiments. The definition of anisotropy is the ratio $r=\left(I_{1}-I_{2}\right) /\left(I_{1}+2 I_{2}\right)$, where $I_{1}$ is the emission or probe signal in a polarization parallel to the excitation and $I_{2}$ that in a perpendicular polarization. One of the classic results of anisotropy theory is that a random array of molecules in which the initially excited states emit with neither rotation nor relaxation to other states exhibits a maximal value $r=0.4$ under steady state excitation. In a time-resolved experiment, this value is the predicted initial value for a the anisotropy due to a transition in any monomeric or dephased multimeric system.

The coherence between eigenstates discussed above can be observed as quantum beats under narrow-line slow dephasing conditions. Fortunately it has another manifestation even in the absence of quantum beats, provided the geometry of the system is appropriate. When the two eigenstates have the same oscillator strength, the initial excited-state anisotropy is predicted $(10 \mathrm{~b}, 15)$ to be 0.7 , in contrast to the well-known limiting value 0.4 . There now exist at least two observed instances of this anomalous initial anisotropy. The first was found in magnesium tetraphenyl porphyrin, a monomer of square symmetry that has two perpendicular degenerate states (15). The second occurs in a genuinely multimeric system, trimers of Cphycocyanin (16), important chromophores of certain photosynthetic organisms. In the case of a two-state system, a full understanding of anisotropy relaxation is far from settled despite a huge literature. I have participated in two recent papers of this topic, wherein further references may be found (17).

Coherence in a somewhat different sense has been predicted and observed in J-aggregate excitons, where it is embodied in a temperature-dependent enhanced or superradiant oscillator strength (18).

\section{Antenna proteins are providing a new variety of delocalized small- $N$ excitons}

Studies of bacterial photosynthesis, for years a thoroughly biochemical domain, have recently uncovered some rather beautiful structures $(8,19)$. They consist of rings of $N$ aromatic chromophores situated in a sufficiently regular environment that the assumption of $\mathrm{C}_{N}$ symmetry appears entirely valid. From an electronic structure standpoint, the rings are essentially cyclo-symmetric versions of the well-known $J-$ aggregate. They support excitations delocalized over several molecules (20) as determined by the properties of transitions between one- and two-exciton states, analogous to the case of $J$ - aggregates (21). These strongly-coupled rings are interesting with respect to not only their electronic structure, but also their possible role in the efficient gathering of energy. Such a role has not been quantified in the case of other strongly coupled pairs and triples of molecules appearing in some photosynthetic arrays. It is speculated (8) that the rings may act as agents for reliable distribution of energy during structural changes; they can transfer wherever they touch another ring or acceptor molecule. However, the major missing ingredient, and challenge for theory, is a determination of an efficient mechanism of insertion and ejection of this energy. 


\section{EQUILIBRATION II: AN OLD THEOREM REVISITED}

\section{The Kennard-Stepanov relation}

Do this thought experiment: optically excite an atomic, molecular, or condensed system with a very short pulse. Then turn off all pathways of decay to the ground state; there is to be no fluorescence, no intersystem crossing, no radiationless decay into the ground manifold. The only processes allowed to remain are relaxations within the vibrational modes of the system and within the surroundings if the system is a solute. Wait for a time sufficient for the system to reach a Boltzmann distribution. (Its zero of energy will be displaced upward by the 0-0 transition energy or the band gap.) Finally, freeze this distribution and allow the system to fluoresce. Measure the fluorescence spectrum and compare it with the absorption spectrum of the system. The ratio of these spectra, frequency by frequency, should reproduce the Wien radiation law with a shifted frequency scale.

Kennard (22) made a computation whose principal assumption is mimicked by the thought experiment described above. He considered the absorption cross section $\sigma(v)$ and emission spectrum $I(v)$ for a general system at temperature $T$ and assumed that emission from the excited state originated in a Boltzmann distribution whose energy scale origin was shifted to the electronic excited level, as described above. Making use of the Einstein A-B relation, he found

$$
F(v) \equiv \log \left[\frac{c^{2}}{8 \pi h v^{3}} \frac{I(v)}{\sigma(v)}\right]=-\frac{h v}{k_{\mathrm{B}} T}+D(T),
$$

where $D(T)$ is a function depending on the energy scale shift and the ratio of the excited and ground state partition functions but not on frequency. This does, indeed, produce the Wien law; stimulated emission has been ignored because fluorescence occurs in a region in which $h v / k_{\mathrm{B}} T \gg 1$. Relation (1) was also developed by Stepanov (23) and is now widely attributed to him. A derivation can be found in one of my current papers (24).

\section{Traditional tests of the relation and a new approach}

The vast generality of the Kennard-Stepanov relation, as I will call Eq. (1), invited many tests of its validity. Traditionally these tests have consisted of plotting the function $F(v)$ from the experimental emission and absorption spectra, estimating its slope, and comparing the temperature deduced from the slope (which I call $T^{*}$ ) with the ambient temperature. Remarkably, first of all, most spectra including the rather complex do produce a nearly straight line for $F(v)$. But secondly, the value of $T^{*}$ is usually not equal to $T$, and is usually somewhat larger. In some cases it has been as large as $2 T$, and it is frequently excitation-energy dependent. Attempts to explain these facts by assuming a "warm" excited state and by assuming an inhomogeneous spectrum have been shown to be inadequate and problematic, respectively (see ref. 25 and citations therein).

A new and more detailed analysis of the experimentally-determined Kennard-Stepanov function $F(v)$ appears to be promising (22). Instead of evaluating the slope of the function as an average over its entire range, we evaluate it locally, producing a frequency-dependent temperature $T^{*}(v)$. This "spectral Kennard-Stepanov temperature" has many remarkable features that we have been able to relate neither to trivial causes such as noise and miscalibration nor to the older hypotheses of warm fluorescence and inhomogeneous broadening. We believe that the several interesting features apparent in $T^{*}(v)$ are indicators of specific relaxation processes, one of which I now describe.

\section{Elementarv models of $T^{*}(v)$ features}

A frequently encountered feature in $T^{*}(v)$ is a peak, resembling any absorption or emission peak. There is a heavy temptation to attribute this to a "hot level," but great caution is necessary. The quantity $T^{*}(v)$ is not yet established as a physical temperature and its position is not directly related to the position of any energy levels. $T^{*}(v)$ relates the slope of a function computed from absorption and emission originating in 
many pairs of levels separated by $h v$ and which may be nearly anywhere within the energy spectrum of the system.

Figure 1 shows the result of using a very simple relaxation model with the theory. We assume that there exist two manifolds of states, each of which involves spectra that would obey the K-S relation if that manifold existed alone. They have average excitation energies differing by $\Delta E$. (The two manifolds might be on the same or separate molecules in solution, or they might be associated with two exciton
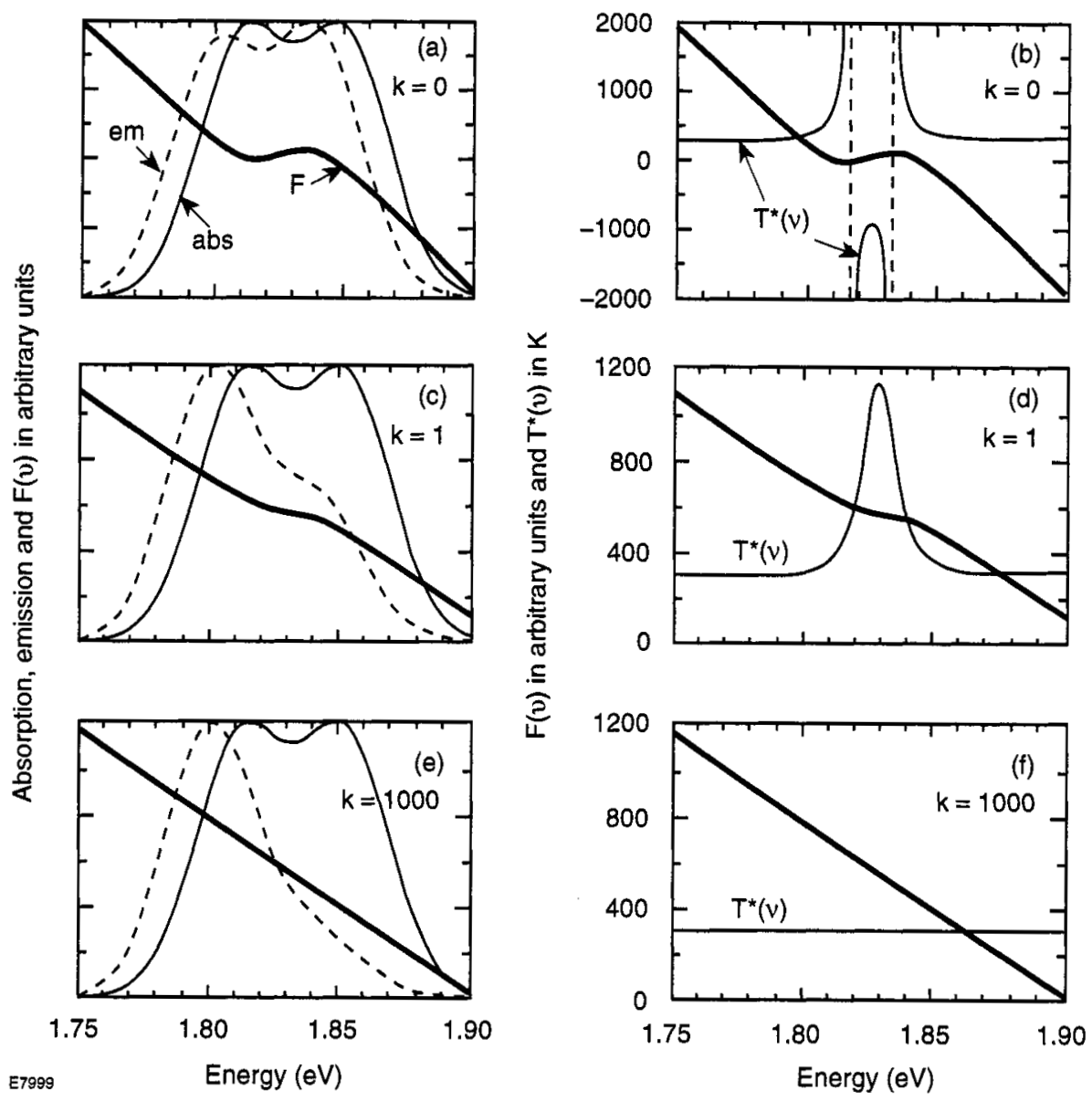

Fig. 1. Results of a model calculation of $F(v)$ and $T^{*}(v)$ for two manifolds interchanging populations with rate constants $k$, downward, and $k \exp \left(-\Delta E / k_{\mathrm{B}} T\right)$, upward. Here $\Delta E$ is the energy difference between the 0-0 transitions of the manifolds. $F(v)$ curves, heavy line, are shown in all parts of the figure, the spectra from which they derive are shown in $(\mathrm{a}, \mathrm{c}, \mathrm{e})$, and $T^{*}(v)$ is shown as indicated in $(\mathrm{b}, \mathrm{d}, \mathrm{f})$. The total oscillator strength is the same in each spectral manifold and is distributed as two resolved Gaussian absorption and emission bands. The absorption FWHM of the individual bands, whose centers are located arbitrarily at 1.810 and $1.851 \mathrm{eV}$, is $17 \mathrm{meV}$. The total absorption and emission are shown as thin solid and dashed lines, respectively, adjusted to full scale. The lifetime of each manifold is taken to be $1.0 \mathrm{~ns}$ and broadband excitation is assumed. As $k$ increases, top to bottom, the kink in the $F(v)$ curve flattens out and $T^{*}(v)$ progresses from divergent (b), to peaked (d), to constant (f). 
bands within the same crystal.) We then assume that the excited-state populations in these two manifolds can interchange energy in accordance with detailed balance, the upper feeding the lower at a rate $k$ and the reverse process occurring at rate $k \exp \left(-\Delta E / k_{\mathrm{B}} T\right)$. The Figure shows how the Kennard-Stepanov relation is recovered at large $k$ relative to the fluorescence rate, and how $T^{*}(v)$ develops a peak and even a singularity as the relaxation rate is decreased. Each of these features has been observed in data we have analyzed .. except for the constant $T^{*}(v)$ that is predicted by the original theory.

\section{Is the K-S relation of use for studving exciton processes in general?}

Although it is too early to give a definitive evaluation of our preliminary $T^{*}(v)$ models, I propose that the unique handling of data connected with the Kennard-Stepanov relation may well be of use in broader contexts in the study of exciton relaxation or equilibration. My research group is looking carefully into the effect of finite relaxation rates within excited manifolds of a homogeneous system and into the more readily tractable case of Förster-coupled excitons in photosynthetic unit models. A limitation for many condensed-matter exciton studies will be the lack of simultaneous accurate absorption and emission data at the same frequencies. However, the original Kennard-Stepanov relation was in fact developed in some detail in a semiconductor context some time ago (26). The new technique described here may suggest further insights for these studies.

The connection between $T^{*}(v)$ and the well-known concepts of hot fluorescence and hot transfer (see, e. g., ref. 27) should be explored. Although, as mentioned above, $T^{*}(v)$ is not to be considered in itself as a true temperature, in a sense it provides a means of projecting out the Boltzmann distribution, or at least amplifying variations around it, through direct reference to experimental data.

\section{SUMMARY AND ACKNOWLEDGEMENTS}

After 65 years, the exciton survives as neither a museum piece for textbooks nor a purely spectrographic detail. It continues to challenge both theorists and experimentalists, it has attained considerable technological significance, and it participates decisively at the very beginning of earth's food chain.

Assistance with graphics and production by the staff of the Laboratory for Laser Energetics, University of Rochester, is greatly appreciated. Computations for Figure 1 were performed by D. A. Sawicki. Research was supported in part by the U. S. Department of Agriculture, NRI Competitive Grants Office project 95-37306-2014, and in part by the National Science Foundation under grants 94-00059 and 94-15583.

\section{REFERENCES}

1. R. S. Knox, Theory of Excitons, Suppl. 5 of Solid State Physics, F. Seitz and D. Turnbull, eds., Academic Press, New York (1963).

2. R. S. Knox, In Collective Excitations in Solids (B. DiBartolo and J. Danks, eds.), pp. 183-225. Plenum Press, New York (1983).

3. R. S. Knox. In Excited State Spectroscopy in Solids (U. M. Grassano and N. Terzi, eds.), pp. 481-509. Il Nuovo Cimento Suppl. (1987).

4. R. S. Knox and W. H. Knox. In Encyclopedia of Applied Physics (G. L. Trigg, ed.), vol. 6, pp. 311-325. VCH Publishers, Inc., New York (1993).

5. Th. Förster, (a) Z. Naturf. 2b, 174-182 (1947); (b) Ann. Phys. (Leipzig) [6] 2, 55-75 (1948).

6. R. van Grondelle, J. P. Dekker, T. Gillbro, and V. Sundström. Biochim. Biophys. Acta 1187, 1-65 (1994).

7. W. Kühlbrandt, D.-N. Wang, and Y. Fujiyoshi. Nature 367, 614-621 (1994).

8. G. McDermott, S. M. Prince, A. A. Freer, A. M. Hawthornthwaite-Lawless, M. Z. Papiz, R. J. Cogdell, and N. W. Isaacs. Nature 374, 517-521 (1995).

9. R. S. Knox. Photosynthesis Research 48, 35-39 (1996).

10. (a) V. M. Kenkre and R. S. Knox. Phys. Rev. Lett. 33, 803-806 (1974); (b) T. S. Rahman, R. S. Knox, and V. M. Kenkre, Chem. Phys. 44, 197-211 (1979).

11. W. H. Knox, J. E. Henry, K. W. Goossen, K. D. Li, B. Tell, D. A. B. Miller, D. S. Chemla, A. C. Gossard, J. English, and S. Schmitt-Rink. IEEE J. Quant. Electronics 25, 2586-2595 (1989).

12. A. Tomita, J. Shah, and R. S. Knox. Phys. Rev. B 53, 10793-10803 (1996).

13. P. D. Laible, W. Zipfel, and T. G. Owens, Biophys. J. 66, 844-860 (1994).

14. R. S. Knox and D. Gülen. Photochem. Photobiol. 57, 40-43 (1993). 
15. C. Galli, K. Wynne, S. M. LeCours, M. J. Therien and R. M. Hochstrasser. Chem. Phys. Lett. 206, 493-499 (1993).

16. M. D. Edington, R. E. Riter, and W. F. Beck. J. Phys. Chem. 99, 15699-15704 (1995).

17. R. S. Knox and R. C. Gilmore, J. Luminescence 63, 163-175 (1995); C. K. Law, R. S. Knox, and J. H. Eberly, Chem. Phys. Lett. 258, 352-355 (1996).

18. F. C. Spano, J. R. Kuklinski, and S. Mukamel. Phys. Rev. Lett. 65, 211-214 (1990).

19. J. Koepke, X. Hu, C. Muenke K. Schulten, and H. Michel. Structures 4, $581-597$ (1996).

20. T. Pullerits, M. Chachisvilis, and V. Sundström. J. Phys. Chem. 100, 10787-10792 (1996).

21. H. Fidder, J. Knoester, and D. A. Wiersma. J. Chem. Phys. 98, 6564-6566 (1993).

22. E. H. Kennard. Phys. Rev. 11, $29-38$ (1918); Phys. Rev. 28, 672-683 (1926).

23. B. I. Stepanov. Sov. Phys. Dokl. 2, 81-84 (1957).

24. D. A. Sawicki and R. S. Knox. Phys. Rev. A, submitted.

25. R. L. Van Metter and R. S. Knox, Chem. Phys. 12, 333-340 (1976).

26. D. E. McCumber. Phys. Rev. 136, A954-A957 (1964); Y. B. Band and D. F. Heller. Phys. Rev. A 38, 1885-1895 (1988).

27. I. Yu. Tehver and V. V. Hizhnyakov. JETP Lett. 19, 191-193 (1974); V. V. Hizhnyakov and I. Tehver. Phys. Stat. Sol. (b) 82, K89-K93 (1977). 